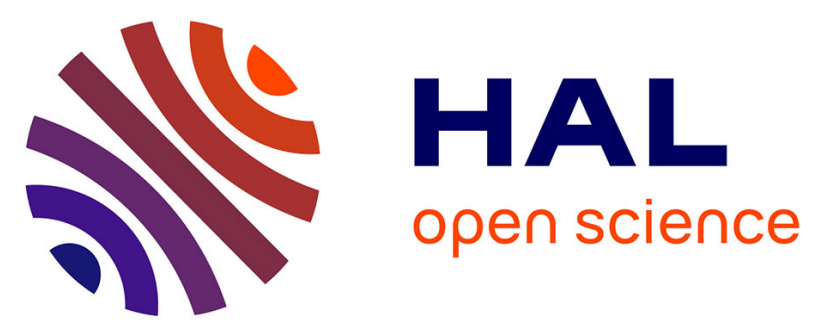

\title{
On the use of satellite-derived frontal metrics in time series analyses of shelf-sea fronts, a study of the Celtic Sea
}

\author{
Lavinia A. Suberg, Peter I. Miller, Russell B. Wynn
}

\section{To cite this version:}

Lavinia A. Suberg, Peter I. Miller, Russell B. Wynn. On the use of satellite-derived frontal metrics in time series analyses of shelf-sea fronts, a study of the Celtic Sea. Deep Sea Research Part I: Oceanographic Research Papers, 2019, 149, pp.103033 -. 10.1016/j.dsr.2019.04.011 . hal-03487964

\section{HAL Id: hal-03487964 \\ https://hal.science/hal-03487964}

Submitted on 20 Dec 2021

HAL is a multi-disciplinary open access archive for the deposit and dissemination of scientific research documents, whether they are published or not. The documents may come from teaching and research institutions in France or abroad, or from public or private research centers.
L'archive ouverte pluridisciplinaire $\mathbf{H A L}$, est destinée au dépôt et à la diffusion de documents scientifiques de niveau recherche, publiés ou non, émanant des établissements d'enseignement et de recherche français ou étrangers, des laboratoires publics ou privés.

\section{(ㄷ)(1) $\$$}

Distributed under a Creative Commons Attribution - NonCommerciall 4.0 International 


\title{
On the use of satellite-derived frontal metrics in time series analyses of shelf-sea fronts, a study of the Celtic Sea
}

\author{
Lavinia A. Suberg ${ }^{\mathrm{a}, *, \#, \text { Peter I. Miller }}{ }^{\mathrm{b}}$, Russell B. Wynn ${ }^{\mathrm{a}}$ \\ ${ }^{a}$ National Oceanography Centre- Southampton, European Way, Southampton, S014 3ZH, \\ UK \\ ${ }^{\mathrm{b}}$ Remote Sensing Group, Plymouth Marine Laboratory, Prospect Place, Plymouth PL1 3DH, \\ UK \\ \# Current Address: Centre Ifremer Bretagne, ZI Pointe du Diable, CS 10070, 29280 Plouzane, \\ France \\ ${ }^{*}$ Corresponding author \\ Email address: 1suberg@ifremer.fr (L.Suberg)
}

Keywords: Frontal metrics, Shelf-sea fronts, Time series analysis, Satellite imagery, Celtic Sea

\section{Author contributions:}

LS and RW developed the concept. Monthly level-4 composites of the various frontal metrics used in the analysis were provided by PM as 8 bit raster files. Data processing and analysis was carried out by LS. Manuscript was written by LS and revised by all authors.

\begin{abstract}
Satellite-derived frontal metrics describe characteristics of oceanic thermal fronts, such as their strength or persistence. They are used in marine science to investigate spatio-temporal variability of thermal fronts or in ecological studies to assist in explaining animal distributions. Although these metrics represent highly processed data, which is based on sometimes complex algorithms, little guidance is available on their correct application in quantitative analyses, in particular for non-specialist users. This research aims to improve accurate use of frontal data. This case study investigates the inter--annual and seasonal variability of two tidal mixing fronts on the Celtic Sea shelf, based on monthly time series of daily frontal maps at $\sim 1 \mathrm{~km}^{2}$ resolution from 1990 to 2010. Some metrics are almost identical and can be grouped, e.g. frontal probability, persistence and so-called "composites" (Pearson correlation: $r=0.8-1.0 ; p<0.001)$, whereas the metric describing frontal strength is distinct from other ones. Consequently, strength and metrics of the frontal probability group showed pronounced differences in their inter-annual and seasonal variability: Strength displayed an oscillating pattern between 1990 and 2010 while there were no significant changes in probability over time. In addition, seasonal variability was affected by segments from adjacent fronts, not belonging to the fronts of interest, which could result in biased estimates.
\end{abstract}


Most important, there was a doubling of available satellite imagery between 1990 and 2010 due to a greater number of operational satellites, which negatively affected frontal probability, positively frontal strength and consequently, changed the temporal pattern of both. When using frontal maps for temporal analyses, we should choose the metric carefully, be aware of biased estimates caused by variability from unwanted frontal segments in the data and account for the variable data quantity. This guide on the use of frontal metrics will be helpful to improve correct interpretations of statistical analyses.

\section{Introduction}

Marine thermal fronts are transition zones in which steep gradients in temperature can be observed over a relatively small distance, often associated with changes in other physical properties, complex hydrodynamics and elevated biomass. Thermal fronts occur over a wide range of spatio-temporal scales, ranging from the large-scale Polar Front to small, short-lived tidal intrusion (Owen, 1981). Frontal metrics derived from remote sensing satellite imagery describe characteristics of these thermal fronts, such as their strength or frequency, in the area of interest and for a desired period. They come in the form of images called frontal maps, which are usually a fusion of multiple satellite images, because single images are often cloudcovered (Miller, 2009). Combining multiple images into one map creates (ideally) a cloud free view on the ocean surface. The resulting frontal maps are a mosaic of pixels containing values describing a front (frontal values) or not (cloud free pixel that cover an area of sea without fronts). The frontal maps provide information on the surface signal of thermal fronts over large spatio-temporal scales, which makes them very popular for scientists from a variety of backgrounds, including oceanographers and ecologists.

Frontal maps are particularly applicable to the study of large-scale processes because of their spatio-temporal coverage: a global and contiguous time series since the 1980's. They have been used to describe their spatio-temporal variability (Hopkins et al. 2010; Lee et al., 2015; Park et al. 2007; Belkin et al., 2009; Nieblas et al. 2014; Oram et al. 2008) and to create maps of surface fronts all over the world (e.g. Canary Upwelling System: Nieto et al., 2012; the Pacific Ocean: Belkin and Cornillon, 2003; Canadian waters: Cry \& Larouche, 2015; California Current System: Armstrong et al., 2012; Indian Ocean: Roa-Pascuali et al. 2015; Japanese Coast: Shimada et al. 2008). Satellite-derived frontal metrics have also become popular in recent years amongst marine ecologists to explain and predict species distributions, particularly for marine apex predators (e.g. Bauer et al. 2015; Nieto et. al 2017; Priede et al. 2009). The potential of fronts to act as biodiversity hotspots has also received attention from 
policymakers involved in development of spatial conservation measures such as Marine Protected Areas (MPAs), and future monitoring of mobile species as part of the Marine Strategy Framework Directive (MSFD) (Defra, 2009;2012; European Union, 2008). Initially, frontal maps were used only descriptively and compared to tracks or distribution maps of marine biota (Doniol-Valcroze et al., 2007; Edwards et al., 2013; McClathie et al. 2012; Wingfield et al. 2011). Now, they are increasingly used in statistical models to investigate bio-physical coupling and ecosystem dynamics (Broodie et al. 2015; Pirotta et al., 2014; Xu et al. 2017).

Frontal metrics represent highly processed data and can be based on complex algorithms, making it difficult for the user to understand the meaning and their limitations when applying statistical analyses, particular for scientist not specialised in the field of remote frontal detection. Although results of quantitative analyses can vary depending on the metric employed, not much guidance for researchers is available in the scientific literature on the use of frontal metrics, the differences between them and factors to consider during their statistical processing. Considering the complex process of generating frontal maps and metrics, this represents essential information for users outside the field to ensure best practice and avoid pitfalls during quantitative analysis.

There is also a lack of information regarding factors influencing the metrics directly, such as the quantity of data used to create a frontal map or the effect of spatial averaging over larger areas in order to create time series. However, it is essential to consider these factors in order to avoid incorrect estimates of a front. For example, there has been a steep and continuous increase in satellite passes over the past 20 years, resulting in an increased number of satellite images per day and therefore, higher data quantity, which affects temporal variability pattern (Oram et al. 2008). Although varying sampling size can affect the results of statistical analyses, not many studies concerning long-term trends of satellite-derived frontal metrics account for this (e.g. Belkin et al., 2005; Kahru et al., 2012; Ullman et al., 2007). Some studies ensure data quality during the processing stage, e.g. only images with at least $90 \%$ cloud-free pixels are used, but do not account for data quantity during statistical analysis (Obenour 2013).

This paper provides guidance on the use frontal metrics and their quantitative analysis, particularly directed towards users outside remote frontal detection. We demonstrate the necessity to account for influencing factors and how to deal with them, including i) a strong non-linear effect of data quantity, ii) bias introduced by not distinguishing between different frontal types and iii) the choice of metric to be used. We show how these factors influence the 
distinct temporal pattern of some commonly used frontal metrics over 20 years from January 1990 to December 2010. The focus of this study are two tidal mixing fronts, which form in the Celtic Sea during the spring when the water is stratified, namely the Celtic Sea and Ushant Front. These two fronts separate the Celtic Sea from the Irish Sea and Western English Channel respectively (Figure 1). Tidal mixing fronts are transition zones between tidally-mixed coastal and seasonally-stratified shelf waters and are critical in shaping oceanographic and biological processes during the summer months (LeFevre, 1986; Simpson and Sharples, 2012). The temporal variability of the Celtic Sea and Ushant Front is well documented from four decades of in-situ and modelling studies (Brown et al., 2003; Elliott et al., 1991; Holt et al., 2010; Neil et al., 2013; Pingree et al., 1978; Young et al., 2004), which provide a reference for the results of this research.

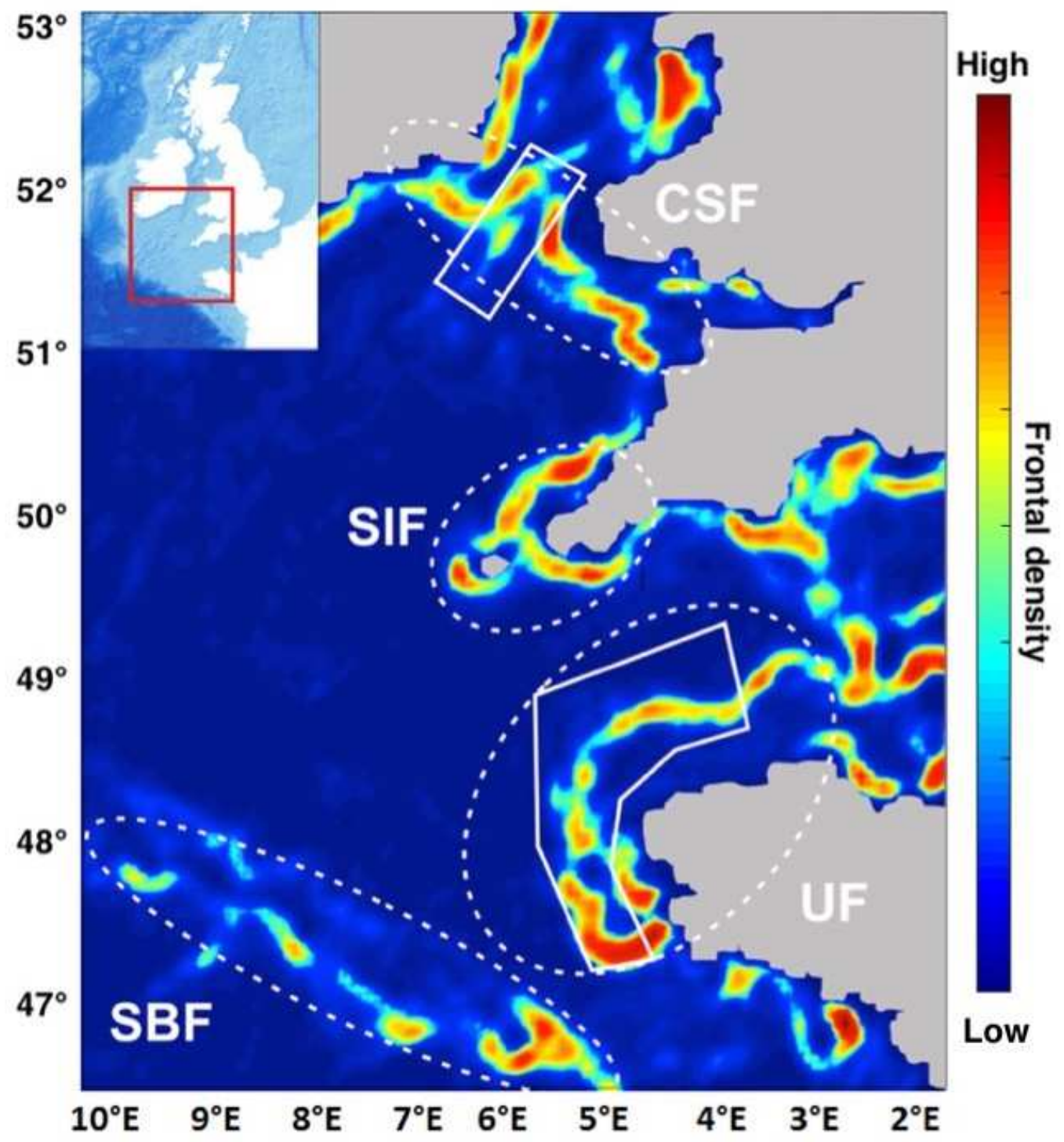


Figure 1 (colour): Average frontal density map (June 2009) showing thermal fronts of the Celtic Sea. Red colours refer to higher frontal density and blue colours to low frontal density. The white dotted circles highlight the tidal mixing fronts UF=Ushant Front, $\mathrm{SIF}=$ Scilly Isles Front, $\mathrm{CSF}=$ Celtic Sea Front and the shelf break front=SBF. The white polygons refer to the two sampling areas used in this research (Celtic Sea and Ushant Front). Parametrisation of the boundary definition for the two front polygons can be found in section 2.1.1 and in the supplement.

\section{Methods}

\subsection{Processing of frontal maps}

Frontal maps used in this research are based on Advanced Very High Resolution Radiometer (AVHRR) data from National Oceanic and Atmospheric Administration (NOAA) satellites from January 1990 to December 2010. The frontal data were processed specifically for this study prior to statistical analysis, using a consistent methodology to produce a multi-decadal time-series for the purpose of exploring the applicability of different front metrics to such analyses. These raw data were acquired, translated into SST values, geo-corrected, cloud masked, and mapped at $1.1 \mathrm{~km}^{2}$ resolution by the NERC Observation Data Acquisition and Analysis Service (NEODAAS) (www.neodaas.ac.uk/data). Both day and night images were considered in order to maximise the detection of fronts in frequently cloud-covered regions: diurnal variations in SST cause negligible effect on front maps because the fronts are detected and their gradients estimated on individual SST scenes rather than on composite maps. In addition, differences are likely to be small, because we were detecting and searching for a particular type of front exactly where it occurs. Fronts were detected on each satellite image by application of the Single Image Edge Detection algorithm (SIED) developed by Cayula and Cornillon (1992). In this approach, a histogram of the SST frequency distribution is created, based on a user-defined array of pixels, but usually 32x32 pixels (also used in this research). If the histogram has a bimodal form, it suggests the presence of two different water masses. In order to qualify as two separate water masses, the temperature difference between the two populations has to be at least $0.4^{\circ} \mathrm{C}$ as recommended when applied to low-noise SST data (Miller, 2009). The SIED then marks the transitional values between the two modes of the histogram as valid pixels $=$ frontal $($ Fvalid $)$.

A SIED-derived frontal map from a single satellite image is unsuitable for the description of meso-scale features due to their high spatio-temporal variability and the frequency of cloud cover in the study region, which disguises dynamic processes (Miller, 2009). Therefore, in this research we aggregated daily front detections into monthly composite maps for each 
metric define below, in order to highlight stable frontal features (Miller, 2009). Although higher temporal resolution would have been more desirable to investigate seasonal pattern of tidal mixing fronts, weekly and fortnightly frontal maps were still highly affected by cloud cover (even during the summer months and particularly at the beginning of the study period in the early 1990's) and were unsuitable for the analysis. In addition, the resolution of the frontal maps was scaled down to $4.8 \mathrm{~km}^{2}$ by taking the mean of a four by four pixel array on the final map. Spatial downscaling was performed to reduce variability around the frontal contours, which facilitated the determination of the sampling area (see supplementary section 8.1). Further steps of data processing depend on the metric chosen and are explained in detail in section 2.2.

\subsubsection{Spatial averaging of frontal pixels over the sampling area}

To investigate inter-annual and seasonal variability of the selected frontal metrics at the Celtic Sea and Ushant Front, a time series for each metric had to be created. For this, all pixels within each of the two frontal areas were spatially averaged to obtain a single value per front and monthly map. We considered all pixels, clear and valid ones in order to avoid bias introduced by variable sample size, e.g. there are more frontal pixels during the summer. The position of tidal mixing fronts varies seasonally, in response to tidal movements, storm events and other factors. Therefore, the sampling area for each front needed to be large enough to capture the spatial variability of the fronts, but small enough to exclude unwanted features in the vicinity as much as possible, which could bias estimates of the fronts of interest (e.g. other fronts such as river plumes or coastal currents). In order to identify a suitable sampling area, core frontal areas were visually identified using Fcomp maps for the Celtic Sea and Ushant Front. Position and extend of each front are known from previous studies (Eliot and Clarke, 1991; Horsburgh et al., 1998; Pingree, 1975; Simpson et al., 1981; Young et al., 2004). Based around the core area, different sized subsets were created, which were resampled to find the most suitable sampling area and to ensure no bias caused by an area size effect was introduced. Details of the resampling approach can be found in the supplement (Section 8.1)

\subsection{Frontal metrics used in this research}

In the following description, the word image refers to a satellite image of the study area, which consists of an array of pixels. Maps refer to the satellite images after frontal algorithms have been applied and show frontal metrics. The example pixel is at a given location of an image (e.g. uppermost left corner), on a map or over a sequence. 
Fclear and Fvalid: For each pixel in the monthly map, Fclear and Fvalid simply provide the total amount of clear and valid pixels respectively. Valid pixels (Fvalid) are pixels that have been identified by the SIED-algorithm as frontal (described in section 2.1). Clear pixels are pixels that were not cloud covered and had a clear satellite view on the ocean, whether or not a front was observed. For example, if 40 images were obtained over the period of one month, 30 of these had clear views on an example pixel, and in the other ten images this pixel was obscured by clouds, the Fclear value for this pixel would be 30 . Out of the 30 clear views, if the example pixel was identified as a front 20 times by the SIED-algorithm, the Fvalid would be 20 .

Fprob (Figure 2 and Table 1) represents the probability of observing a front in a given pixel over the sequence of images used (Miller, 2009). As in the example above, out of the 30 clear views, if the example pixel was identified as a front 20 times by the SIED-algorithm, then the Fprob value for this pixel would be:

$$
\text { Fprob }=\frac{\text { front pixels }}{\text { clear pixels }}=\frac{20}{30}=0.67
$$

Frontal pixels (also called valid pixels) and clear pixels are described in more detail further below under Fvalid and Fclear. The higher the Fprob value, the more often a front was detected in the pixel. Therefore, clusters of pixels with high Fprob on a frontal map represent areas of higher frontal occurrences. The advantage of Fprob is that it is simple and easy to understand. However, there are two apparent disadvantages. Firstly, it is a proportion and can easily be biased when the relationship between the numerator and denominator is not linear or if both change in the same direction, but at different rates. Secondly, Fprob does not provide information on the strength of a front.

Fmean provides information on the temperature gradient (temperature change per distance of pixel resolution, in this case ${ }^{\circ} \mathrm{C} / 4.8 \mathrm{~km}^{2}$ ) and hence, an indication of the strength of a front (Miller, 2009). After applying the SIED-algorithm to a single image, the temperature gradients between a front pixel and its neighbouring pixels are calculated. The value of the greatest gradient found is assigned to the example pixel. This is done for all valid pixels on a map and all images going into a map. For the monthly map, the mean of all temperature gradient values is calculated for the example pixel. However, the mean is only based on front pixels in the sequence and not on pixels that were cloud free but non-frontal as it is the case for Fprob. This is in order to avoid degrading the metric with gradients not associated with fronts, or with low gradients observed where a dynamic front was previously located. Using 
the same example as above, the temperature gradient was calculated for the 20 front observations of the example pixel.:

$$
\text { Fmean }=\frac{\text { sum of gradient values }(20 \text { different values })}{\text { total number of frontal pixels }}=(\text { e.g. }) \frac{21.4}{20}=1.07
$$

It should be noted that Fmean disregards of clear pixels. One the one hand, this makes Fmean less sensitive to data quantity (Fclear) and does lessen the visualisation of ephemeral features. On the other hand, it does not distinguish between pixels that were identified as frontal frequently versus ones that were not. For instance, the example pixel was identified as frontal 20 times in the sequence of 30 clear images and had an Fmean of 1.07. Another pixel has been identified as frontal twice in the sequence of 30 clear images, but also had a temperature gradient of 1.07 each time. This pixel will receive the same value on the map as first one although its frontal frequency was very small. This results in maps containing many transient frontal segments that are displayed with the same strength as the persistent ones, which can introduce noise to a map.

Fpers is the product of multiplying the final (in our case monthly) map of Fmean by the final map of Fprob:

$$
\text { Fpers }_{\text {final }}=\text { Fmean }_{\text {final }} \times \text { Fprob }_{\text {final }}
$$

By weighting Fmean by a measure of persistence (Fprob), areas of frequently occurring fronts are highlighted and noise introduced by short-lived frontal segments is reduced (Miller, 2009). While the multiplication of Fprob and Fmean aids visualisation of more consistent features, it complicates interpretation of the metric itself, because it is comprised of two entities that have different meanings. A change in Fpers cannot be directly attributed to either changes in Fprob or Fmean (or both), whereas it might be crucial to know which metric is more affected, e.g. if interested in the meteorological drivers of the observed variability.

In Fcomp maps an additional weighting factor is applied to the monthly map of Fpers, which considers the spatial proximity of frontal pixels (Miller, 2009):

$$
\text { Fcomp }_{\text {final }}=\text { Fpers }_{\text {final }} \times \text { weighting factor }
$$


Pixels near or in clusters of valid pixels, will receive an additional boost. The closer the pixel is to a frontal cluster, the more it will be boosted. This process will ignore pixels located beyond a certain distance from any frontal clusters. The resulting maps further emphasise persistent features and further reduce the occurrence of noise. Like Fpers, Fcomp obscures the influence of each of the components for the final product and it is not possible to identify the most variable component.

Fdens is an Fcomp map with an additional spatial smoother (in this case a Gaussian filter of five pixels width) applied to the final Fcomp map in order to turn the discrete front segments into a continuously varying spatial map (Scales et al., 2015). Fdens is particularly useful for visualisation of persistent, spatially stable features as it removes nearly all transient frontal segments:

$$
\text { Fdens }_{\text {final }}=\text { Fcomp }_{\text {final }} \times \text { spatial smoother }
$$

Table 1: List of metrics used in this research and their abbreviations, common names, quantitative derivation and value range. All are at monthly $4.8 \mathrm{~km}^{2}$ resolution

\begin{tabular}{|c|c|c|c|}
\hline Metric & Common name & Definition & Value range \\
\hline Fvalid & Valid pixels & $\begin{array}{l}\text { Total of valid (frontal) pixels in a } \\
\text { sequence of images }\end{array}$ & $\begin{array}{l}\text { Any positive } \\
\text { integer }\end{array}$ \\
\hline Fclear & Clear pixels & $\begin{array}{l}\text { Total of clear pixels in a sequence of } \\
\text { images }\end{array}$ & $\begin{array}{l}\text { Any positive } \\
\text { integer }\end{array}$ \\
\hline Fprob & Frontal probability & $\frac{\text { Fvalid }}{\text { Fclear }}$ & $0-1$ \\
\hline Fmean & $\begin{array}{l}\text { Temperature } \\
\text { gradient }\end{array}$ & $\frac{\text { Temperature gradient }}{\text { Fvalid }}$ & $0-2.54$ \\
\hline Fpers & Frontal persistence & Fprob $\times$ Fmean & $0-0.254$ \\
\hline Fcomp & Frontal composite & $\begin{array}{l}\text { Fpers } \times \text { Fprox } \\
\text { Fprox }=\text { additional boost, when other } \\
\text { frontal clusters in the neighbourhood }\end{array}$ & $0-0.254$ \\
\hline Fdens & Frontal density & Fcomp + spatial smoother & $0-0.254$ \\
\hline
\end{tabular}



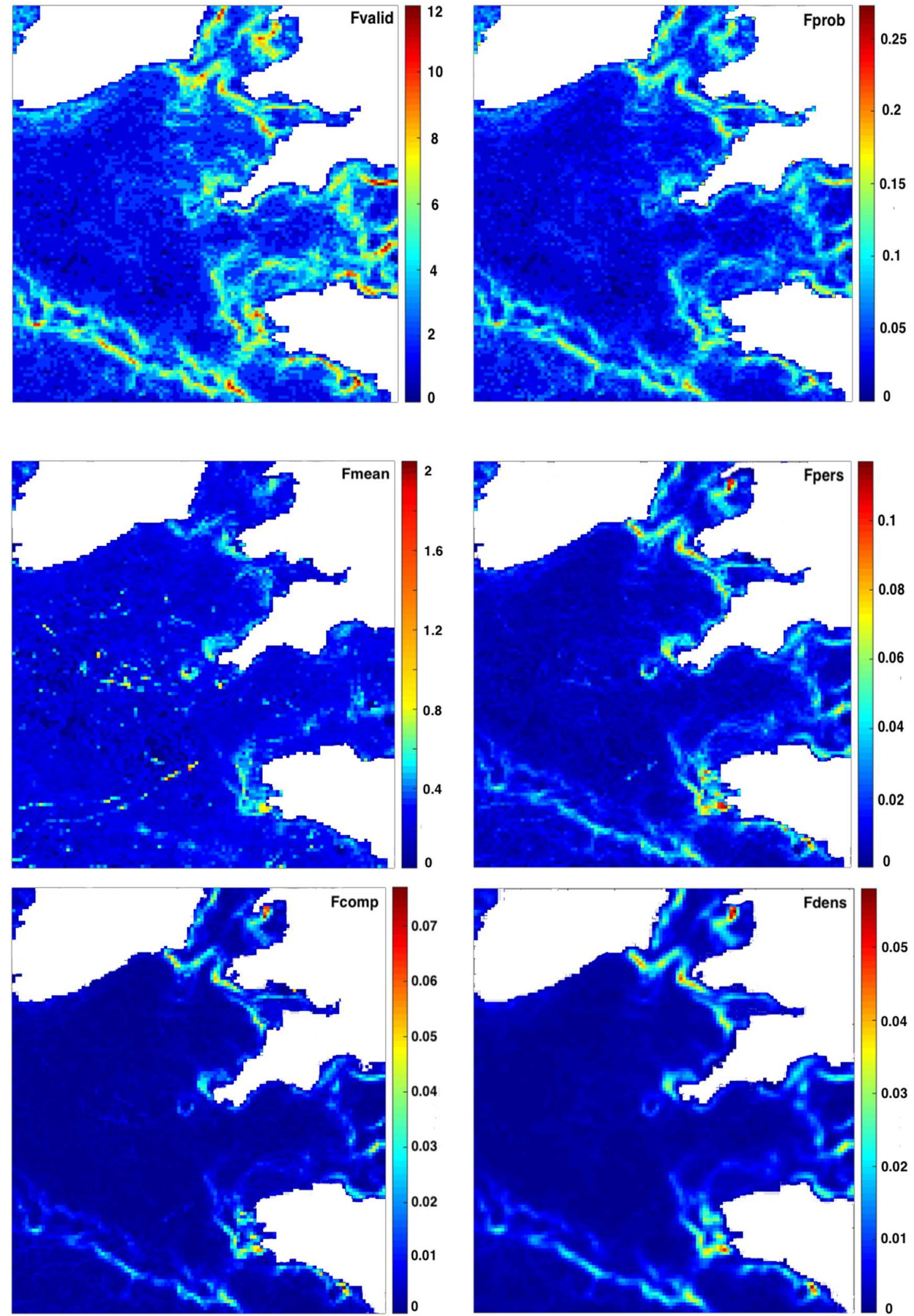

Figure 2 (colour): Monthly maps for Fvalid, Fprob, Fmean, Fpers, Fcomp and Fdens from June 2009. Pixels covering land are no-value pixels and therefore, come up as white. 


\subsection{Statistical analyses}

Correlation analyses showed that the metrics Fprob, Fpers, Fcomp were strongly related. Fdens displayed highest correlations with Fcomp and Fmean (Table 2). Subsequently, analyses in this research were conducted on Fprob (representative for the group Fprob, Fcomp and Fpers) and Fmean only. Fprob was selected because it is a) more comprehensible than other complex metrics, b) frequently used in remote sensing research, and c) the driving component in Fcomp and Fpers in our dataset (although this can differ in other systems, e.g. California Current System, Nieto et al. 2012). Fmean has been less frequently used in ecological or oceanographic time series, but is included because it provides useful information on the strength of the front and hence, other characteristics than Fprob.

Table 2: Pearson Product Moment correlation coefficients $(r)$ after extraction of the seasonal variability for all metrics combinations. Lower left diagonal (blue font) refers to Celtic Sea Front and upper right diagonal (black front) to Ushant Front correlations. Coefficients above 0.7 are in bold and, italic numbers are coefficients of correlation analyses with $p$-values $<0.05$.

\begin{tabular}{llllll}
\hline Metric/r & Fprob & Fpers & Fcomp & Fmean & Fdens \\
\hline Fprob & - & $\mathbf{0 . 9}$ & $\mathbf{0 . 9}$ & -0.04 & 0.3 \\
Fpers & $\mathbf{0 . 9}$ & - & $\mathbf{1 . 0}$ & 0.2 & 0.5 \\
Fcomp & $\mathbf{0 . 9}$ & $\mathbf{1 . 0}$ & - & 0.2 & 0.6 \\
Fmean & -0.3 & 0.06 & 0.06 & - & 0.6 \\
Fdens & 0.3 & 0.5 & 0.6 & 0.6 & - \\
\hline
\end{tabular}

Inter-annual and seasonal variability of Fprob and Fmean and the effect of Fclear on this variability were investigated using anomalies. Anomalies for statistical analysis were created by subtracting the overall mean of the time series from each data point of the time series (each month-year combination). Temporal explanatory variables were year to account for interannual variability, month to account for seasonal variability and Fclear to account for variations in data quantity. To demonstrate the effect of Fclear on Fprob and Fmean, predictions of monthly and yearly variability of the two metrics are shown from two models, one with and one without the Fclear variable. For visualisation purposes, monthly and yearly anomalies were calculated by subtracting the overall mean from the mean of each month/year respectively. For inter-annual variability plots only months March to November were considered (see below) to avoid the unwanted inclusion of wintertime fronts (present in the study area) not associated with the tidal mixing fronts.

Generalized Additive Mixed Models (GAMMs) with an autoregressive correlation structure of order one (AR(1)) were used in order to account for temporal autocorrelation and the nonlinear relationship between the response and explanatory variables. The GAMMs take the 
structure as specified by Hastie and Tibshirani (1987) and were fitted using the gamm function in the $m g c v$ package (Wood, 2006). Smoothed terms were fitted as regression splines with fixed maximum degrees of freedom $(k=6)$ for the covariate month and Fclear in order to avoid overfitting. The variable month was modelled using cyclic cubic regression splines, setting knots manually between 3 (March) and 11 (November) in order to account for the circular nature of this term. Model selection was conducted using manual stepwise-backwards selection. Model fit was examined by means of residual analysis. Residual analysis displayed a few single outliers in the Celtic Fprob model. The outliers were excluded and the model rerun, which improved model fit, but did not affect significances of the variables.

\section{Results}

\subsection{Temporal variability of Fprob and Fmean}

Due to the distinct nature of the two metrics, their temporal patterns differed significantly. Fprob anomalies were positive until 1996 and dropped sharply thereafter at both fronts. Apart from minor variations, temporal variability of Fprob was consistent for the remainder of the time series. Extremely high values of Fprob were observed in 1990 and 1996 at the Celtic Sea Front, which were less pronounced at the Ushant Front. In contrast to Fprob, Fmean displayed temporal fluctuations with an initial decrease from 1990 to 1996, followed by an increase from 1997 to 2010 at both fronts (Figure 3). A notable low in Fmean occurred in 1996 at the Celtic Sea and Ushant Front. Overall differences between the Celtic Sea and Ushant Front were low for each metric and occurred predominantly in the first ten years of the time series. In addition, values for both metrics were slightly higher at the Celtic Front compared to the Ushant Front: Fprob Celtic: 0.078 \pm 0.03 , Ushant: 0.072 \pm 0.03 ; Fmean Celtic: $0.22 \pm 0.09$, Ushant: $0.19 \pm 0.08 ;$ ).

There was a fairly consistent increase in Fclear and Fvalid from 1990 to 2010 (Figure 3). Anomalies became positive at both fronts in the middle of the time series, around 2001. However, since 2005 the trend stagnated and there was even a slight decrease in Fclear and Fvalid in the late 2000's. Notable lows in Fclear and Fvalid coincided with the high Fprob and low Fmean years of 1990 and 1996. The relationship between the observed increase in Fclear and interannual variability of Fprob and Fmean is described in the following section 3.2.

Seasonal patterns for Fprob differed between the Celtic Sea and Ushant Front (Figure 4). Fprob values at the Ushant Front were decreasing until April, became positive in June and did 
not drop to negative until December. At the Celtic Sea Front, seasonal fluctuations of Fprob were more variable. Anomalies were positive during the summer from June to September, negative between October and November, positive again until February and again negative until June (Figure 4). The positive Fprob anomalies during the winter months, when tidal mixing fronts are absent, indicate the inclusion of frontal segments that are not the focus of this study. In this case, this unwanted signal was likely introduced by parts of a coastal current that runs along the east coast of Ireland. By restricting the sampling subset to $12 \mathrm{~km}$ away from the coasts, it was anticipated to exclude coastal influences, which was clearly not sufficient. Fmean displayed a typical seasonal curve at both fronts with increasing values from the beginning of the year until August/September and a sharp decrease thereafter.

Fclear and Fvalid exhibited typical seasonal cycles, similar to the one seen for Fmean (Figure 4). Positive anomalies of Fvalid occurred from May to September at the Celtic Sea Front and May to October at the Ushant Front. Anomalies of Fclear were positive throughout March to September at both fronts. However, Fclear values dropped notably in July and increased slightly again thereafter. 

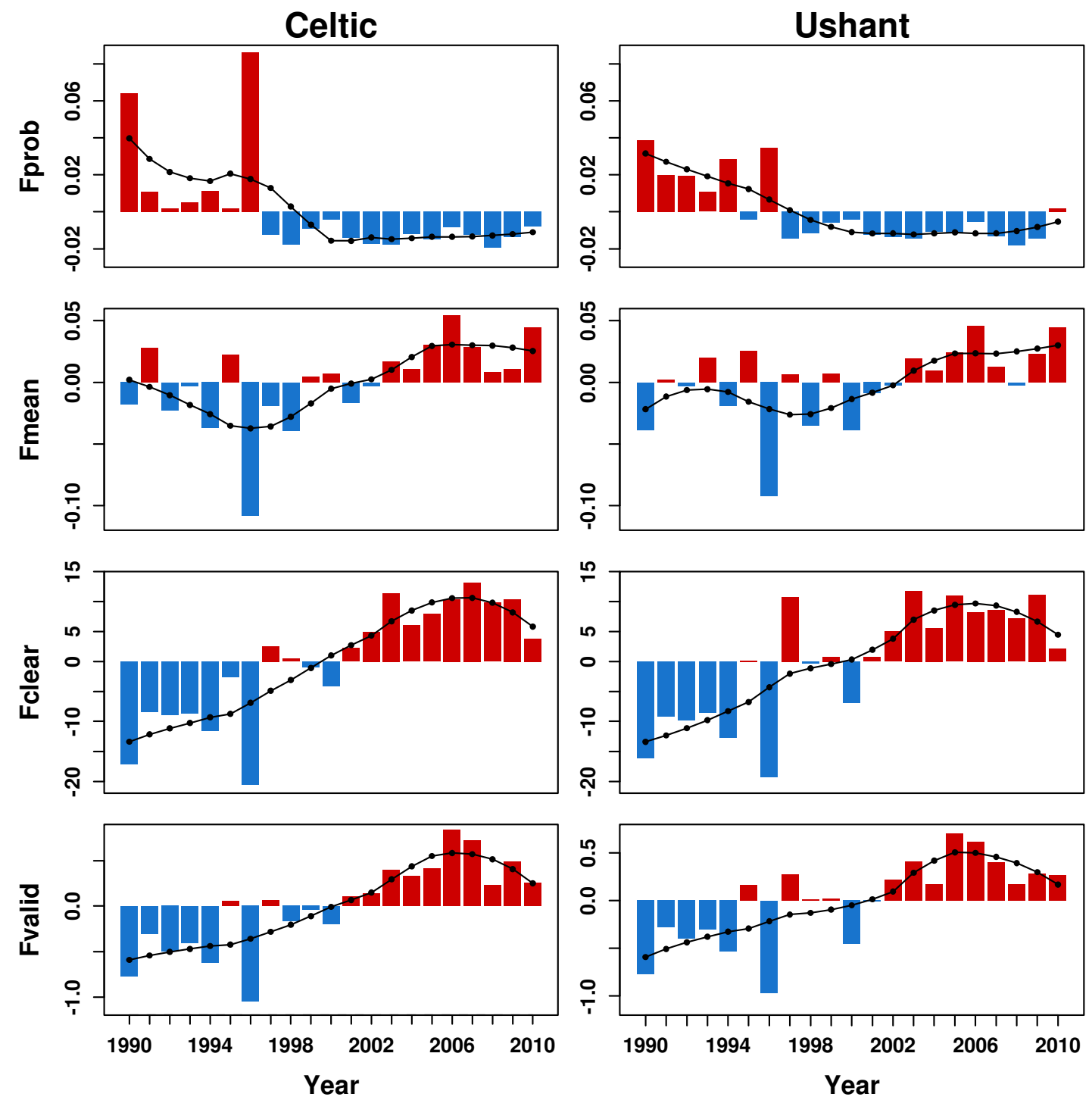

Figure 3 (colour): Yearly anomalies of Fprob, Fmean, Flcear and Fvalid at the Celtic Sea and Ushant Front from 1990 to 2010. Anomalies are based on a seasonal subset (March to November). Blue bars represent negative anomalies and red positive anomalies. Black line represents loess smoother $(\alpha=0.6)$. 

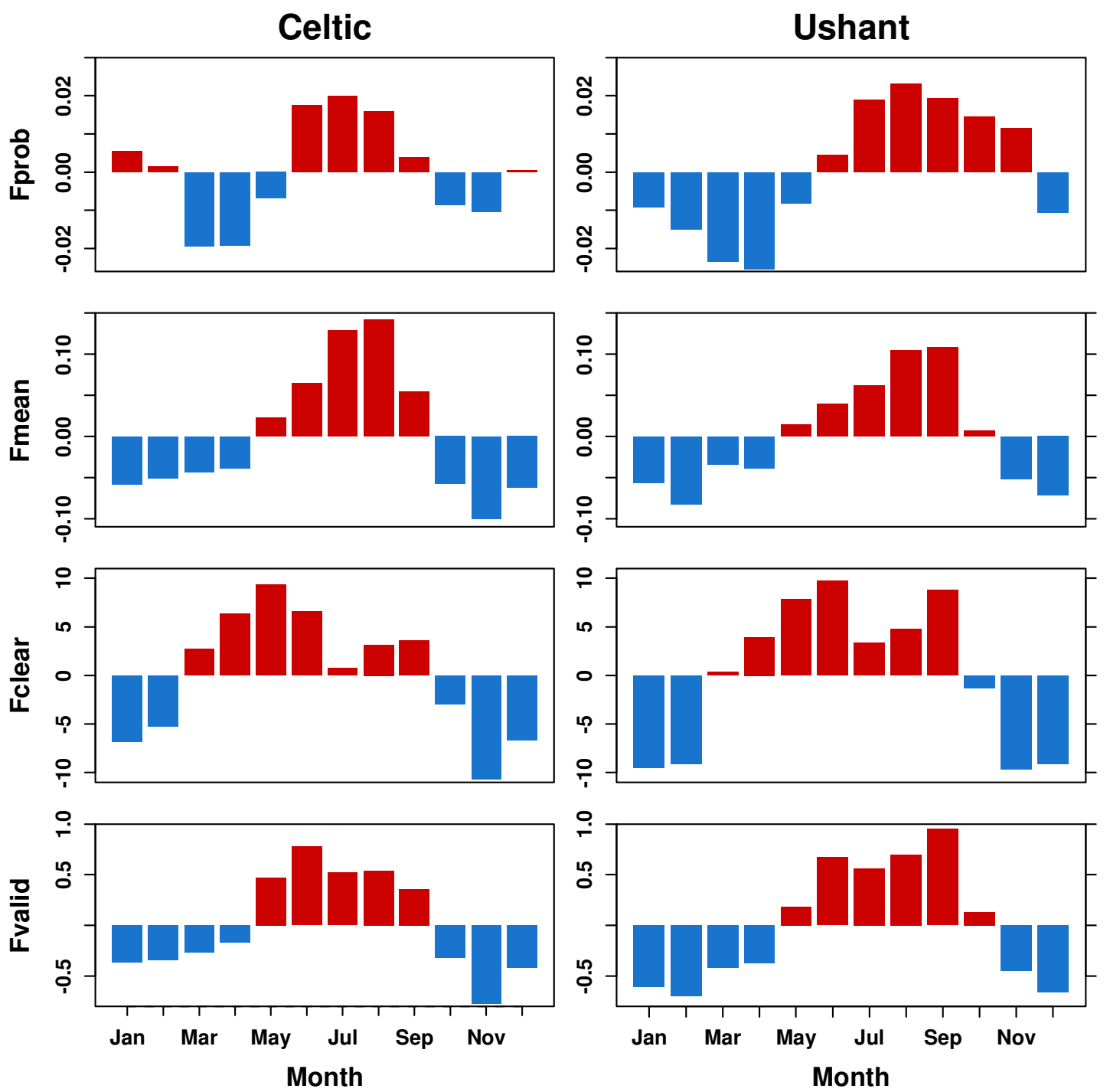

Figure 4 (colour): Monthly anomalies (based on the entire time series) for of Fprob, Fmean, Flcear and Fvalid at the Celtic Sea and Ushant Front. Blue bars represent negative anomalies and red positive anomalies.

\subsection{Effect of Fclear on variability of Fprob and Fmean}

Preliminary analyses indicated a correlation between Fclear and the two metrics Fprob and Fmean. The temporal pattern seen for Fprob and Fmean might not purely be a result of changes in meteorological or hydrodynamic forcing over seasonal and interaannual cycles, but caused to a certain degree by variations in available data. To investigate an effect of Fclear on temporal variability of Fprob and Fmean, inter-annual and seasonal variability of both metrics were modelled including Fclear as an explanatory variable. In a follow up analysis, which is not presented here, temporal variability of these fronts was investigated in relation to meteorological factors known to influence frontal dynamics (e.g. heat flux, wind speed), but which are also partly correlated with Fclear (Suberg, 2015). However, an Fclear 
effect remained even when accounting for atmospheric forcing and can therefore, not be explained by covariability with meteorological factors alone. For brevity purposes, this analysis focuses on Fclear only.

There was also a significant effect of Fclear on Fprob (Figure 5 and Table 3). The relationship was negative and levelled off at higher Fclear values (Figure 5). The inclusion of Fclear caused a notable modification of the interannual pattern of Fprob. The model accounting for Fclear did not suggest significant interannual variability in Fprob at the Celtic Sea and Ushant Front, whereas a model without Fclear suggests a negative trend over time (Figure 5, red lines). In addition, the seasonal curve of Fprob was more distinct when accounting for Fclear and showed the expected pattern with higher Fprob values in summer and lower values during the winter, when tidal mixing fronts are absent.

The relationship between Fclear and Fmean at both fronts was very strong and overall, positive (Figure 6 and Table 3). The relationship was stronger at the lower value range of Fclear and levelled off with increasing Fclear (Figure 6). In consequence, accounting for Fclear resulted in changes in the interannual pattern of Fmean. The decrease at the beginning of the time series was stronger and the increase in the second half was less steep compared to the pattern seen in Figure 3. When Fclear was not included in the model, the relationship between Fmean and time was positively linear (Fig. 6, red lines). Although the model fit should be interpreted with caution as it appears to be an oversimplification of the real relationship. Seasonal variability on the other hand, was not greatly affected by Fclear and still displayed the seasonal cycle and timing as seen in Figure 4. While factors Fclear and months explained considerable amount of the variability, year only lead to a 0.03/0.04 (Celtic Sea/Ushant) increase in the model $\mathrm{R}^{2}$ (Table 3). A summary of the effect of Fclear on temporal variability of Fprob and Fmean is given in Table 4.

Table 3: Summary of GAMMs with AR1 structure for a seasonal subset of Fprob and Fmean (March/April to November) anomalies for Celtic Sea and Ushant Front modelled as a function of year, month and Fclear (coefficients for model including Fclear shown in black, model without Fclear shown in red). Only significant covariates are listed, including their estimated degrees of freedom (edf), F-values, p-values and reduction in AIC. The adjusted $\mathrm{R}^{2}$ for the final model is given in bold (Adj. $\mathrm{R}^{2}$ ) and increase for each additional variable.

\begin{tabular}{lllllll}
\hline Metric & Front & Covariate (edf) & F-value & p-value & $\Delta$-AIC & Adj. $\mathbf{R}^{2}$ \\
\hline & Celtic & Month $(3.82 ; 3.3)$ & $36.1 ; 10.5$ & $<0.001 ;<0.001$ & $108.93 ; 25.6$ & $0.2 ; 0.2$ \\
Fprob & Front & Fclear (6.82) & 33.65 & $<0.001$ & 156.98 & 0.81 \\
& & Year (1.4) & 13.1 & $<0.001$ & 11.2 & $\mathbf{0 . 4}$
\end{tabular}




\begin{tabular}{lllllll} 
& Ushant & Month $(3.54 ; 2.9)$ & $26.03 ; 7.7$ & $<0.001 ;<0.001$ & $48.72 ; 15.7$ & $0.18 ; 0.2$ \\
& Front & Fclear $(4.47)$ & 27.58 & $<0.001$ & 60.05 & $\mathbf{0 . 5 9}$ \\
& & Year $(1.9)$ & 10.7 & $<0.001$ & 11.7 & $\mathbf{0 . 4}$ \\
\hline & Celtic & Year $(2.77 ; 1.0)$ & $4.85 ; 8.5$ & $0.004 ; 0.004$ & $4.33 ; 3.6$ & $0.03 ; 0.03$ \\
& Front & Month $(3.85 ; 3.8)$ & $99.96 ; 68.3$ & $<0.001 ;<0.001$ & $167.0 ; 137$ & $0.69 ; \mathbf{0 . 6 8}$ \\
Fmean & & Fclear $(4.21)$ & 24.67 & $<0.001$ & 67.16 & $\mathbf{0 . 8 2}$ \\
& & & & & \\
& & & & & \\
& Ushant & Year $(4.27 ; 1.0)$ & $4.27 ; 9.5$ & $<0.001 ; 0.002$ & $17.54 ; 4.7$ & $0.04 ; 0.03$ \\
& Front & Month $(3.66 ; 3.7)$ & $67.5 ; 40.1$ & $<0.001 ;<0.001$ & $103.82 ; 86.8$ & $0.53 ; \mathbf{0 . 5 3}$ \\
& & Fclear $(4.26)$ & 47.09 & $<0.001$ & 11.9 & $\mathbf{0 . 7 8}$ \\
\hline
\end{tabular}
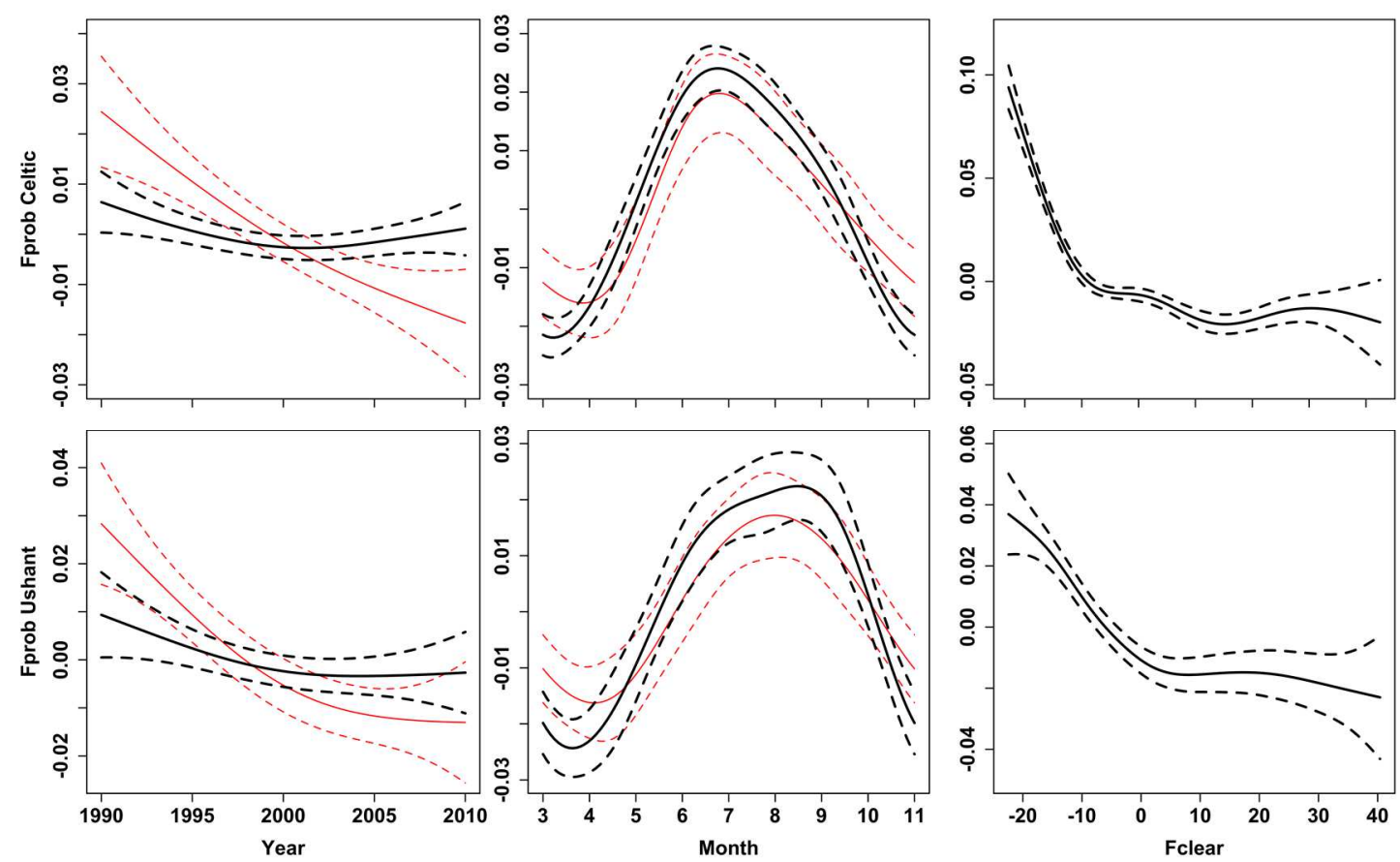

Figure 5: GAMM predictions showing temporal variability (year and month) of Fprob anomalies with (black) and without (red) accounting for Fclear and the relationship between Fprob and Fclear. An AR1 structure was added to the GAMM to account for temporal autocorrelation. The model is based on a seasonal subset of Fprob (March/April to November, $N=189 / 168)$. Upper panel shows Celtic Sea Front, lower panel Ushant Front. Solid lines represents fitted values, dotted lines 95\% confidence intervals. Note: factor "year" was insignificant for the inclusive Fclear model (black lines) and is not shown in table 3. 

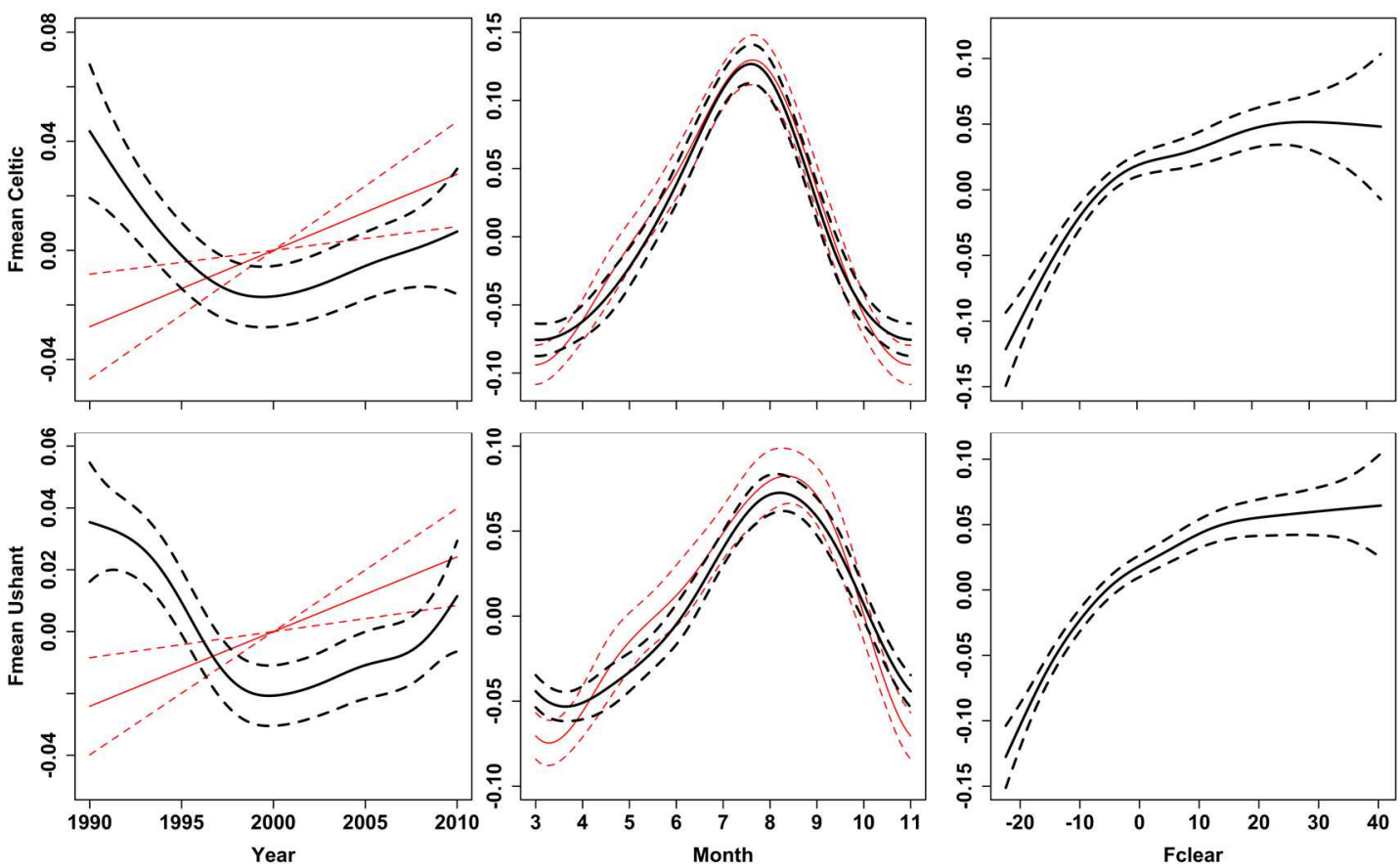

Figure 6: GAMM predictions showing temporal variability (year and month) of Fmean anomalies with (black) and without (red) accounting for Fclear and the relationship between Fmean and Fclear at the Celtic Sea Front and Ushant Front. An AR1 structure was added to the GAMM to account for temporal autocorrelation. The model is based on a seasonal subset of Fmean (March/April to November, $N=189 / 168$ ). Upper panel shows Celtic Sea Front, lower panel Ushant Front. Solid lines represents fitted values, dotted lines 95\% confidence intervals.

Table 4: Summary table of the significance of the number of clear pixels and its effect on inter-annual and seasonal variability of Fmean and Fprob at both fronts Celtic Sea and Ushant Front.

\begin{tabular}{lll}
\hline Metric & Front & Effect of Fclear \\
\hline \multirow{3}{*}{ Fprob } & Celtic Front & $\begin{array}{l}\text { Significance: Yes (negative correlation) } \\
\text { Inter-annual variability: Strong effect } \\
\text { Seasonal variability: Weak effect }\end{array}$ \\
& & Significance: Yes (negative correlation) \\
& Inter-annual variability: Strong effect \\
& Seasonal variability: Weak effect \\
& & \\
\hline \multirow{3}{*}{ Fmean } & & Significance: Yes (positive correlation) \\
& & Inter-annual variability: Strong effect \\
& & Seasonal variability: Weak effect \\
& & \\
& & Significance: Yes (positive correlation) \\
& & Inter-annual variability: Strong effect \\
& & Seasonal variability: Weak effect \\
\hline
\end{tabular}




\section{Discussion}

This research uses time-series analyses of two seasonal shelf-sea fronts as a framework for the first coherent guide on the use of satellite-derived frontal metrics in quantitative analyses. The results of the study will be discussed in the context of managing frontal metrics in quantitative analyses.

\subsection{Recommendations on the metric for temporal analyses}

Fprob and Fmean describe two distinct characteristics of a front (probability versus strength) and consequently, display specific and independent temporal pattern. It is important to keep in mind that the two metrics are complementary and both are required in order to describe a frontal feature thoroughly. Therefore, we recommend the combined use of both metrics to investigate differences in temporal pattern and relationships with other (e.g. environmental) variables for each metric.

Results of this study concur with previous research and support the suitability of Fprob and Fmean for temporal variability studies. The seasonal cycles of Fprob and Fmean are in agreement with the onset and breakdown of stratification in the Celtic Sea and previous observations of the Celtic Sea and Ushant Fronts (Eliot and Clarke, 1991; Pingree, 1975; Young et al., 2004). Model simulations of stratification in the Celtic Sea predict the thermocline to establish around the Celtic Deep first (near the Celtic Sea Front) around April, advancing over the shelf and reaching the Western English Channel (location Ushant Front) within a month. The delay in frontal development between the Ushant and Celtic Sea Front was also indicated by the satellite data (Figure 4, 5 and 6). Interesting to note is furthermore, the seasonal curve for Fmean being slightly sharper than the one for Fprob due to the effect of stratification on Fmean. Once the fronts are established (June to August for the Celtic and July to September for the Ushant Front), frontal probability remains fairly stable, whereas the frontal strength consistently changes in response to decreasing and increasing temperatures/stratification.

Inter-annual pattern of Fprob showed abnormally high values (and low values in Fmean) in 1990 and 1996. These extremes are partially caused by confounding factors, such as higher than usual cloud cover, which led to a reduction of available satellite imagery. Other explanations will be discussed in the next section (4.2). Apart from these extremes, no obvious changes in Fprob occurred over the study period. The results of the long-term 
analysis suggest that the strength of the frontal temperature gradient (Fmean) oscillated between 1990 and 2010 at both fronts (Figure 6). Oscillations in frontal strength are expected in response to meteorological forcing (Holt et al, 2010). In a follow up analysis, which investigates the underlying drivers of the observed temporal variability, SST and net heat flux were found to be the predominant meteorological factors explaining the variation in Fmean (Suberg, 2015). An increase in SST in the study area could have caused the observed intensification of Fmean over the later ten years of the time series. Modelling studies predict tidal mixing fronts in the Celtic Sea to intensify due to increasing water temperatures during this century (Holt et al, 2010; Marsh et al., 2015).

Fcomp, Fpers or Fdens were not analysed in detail here to their high correlation with Fprob and/or Fmean. This is essentially due to the fact that Fprob and Fmean are base metrics for describing frontal characteristics and all other metrics are derivates of either one or both. In general, we recommend the use of Fprob and Fmean for temporal analysis over Fcomp, Fpers or Fdens, because the later complicate interpretation without providing additional information. Fpers could serve as a synthesis of both Fmean and Fprob, but with some restrictions as it can be dominated by either one of the two components. Fcomp and Fdens represent slightly more contrasted versions of Fpers and are quite suitable for visualization purposes or a synthetic spatial analysis, because they allow for clearer distinction between low and high frontal frequency areas.

\subsection{Effect of data quantity on frontal metrics}

Fclear had significant, but contrasting effects on the temporal pattern of Fprob and Fmean. Overall, the relationship between Fclear and Fmean was positive, but levelled out at high numbers of clear pixels. More clear pixels will lead to more cloud free scenes and subsequently, a higher detection rate of frontal segments. In addition, indirect factors increase the relationship between Fmean and Fclear. Stronger temperature gradients across tidal mixing fronts are likely to be correlated with summer months or good weather periods with less cloud cover, stronger solar irradiance and higher temperatures. Under these conditions, tidal mixing fronts will strengthen or develop quicker (Holt et al., 2010; Young et al., 2004). At the same time, summer months and decreased cloud cover are also linked to higher Fclear. Therefore, it is essential to account for data quantity when using Fmean for quantitative analyses. Fmean has not been widely used in time series analysis and comparisons with other studies are not possible. 
In contrast to Fmean, the relationship between Fprob and Fclear in the lower value ranges was negative. The reason for the negative correlation is that Fprob is a simple proportion between valid and clear pixels (Fvalid and Fclear). There was a strong positive correlation between Fvalid and Fclear ( $r=0.8$ ) and a notable increase over time for both. In addition, years with notably low Fclear, and for that matter low Fvalid (e.g. 1990 and 1996), showed disproportionally high Fprob values. This contradictive pattern is due to a divisor effect. Over the time frame of this research, the increase in number of satellites has led to an increase in the number of clear pixels (Fclear), which was much higher than the increase in the number of front pixels (Fvalid). For example, from the first five years of the time series (1990-1994) the average number of front pixels in a given location (pixel) increased from $0.97 \pm 0.42$ to $1.91 \pm 0.86$ in the last five years (1996-2010) at the Celtic Sea Front (Ushant: from $0.88 \pm 0.45$ to $1.56 \pm 0.9$ ), whereas clear pixels have risen from $11.62 \pm 6.15$ to $30.75 \pm 13.38$ (Ushant: from $10.7 \pm 6.55$ to $27.28 \pm 15.22$ ). This represents a 2.65 -fold increase in clear pixels (Ushant: 2.55 ), but only a 1.97 -fold increase in front pixels (Ushant: 1.77). Therefore, the number of front pixels is divided by an increasingly higher number of clear pixels over time, which results in a decrease of Fprob (Fprob $=$ Fvalid/Fclear). The average Fprob for 1990-1994 was 0.08 compared to 0.06 between 2006 and 2010 at both fronts. According to this, frontal probability has decreased by $25 \%$ from the first to the last quarter of the time series, which is unlikely and not supported by any other studies concerning interannual variably of Fprob (e.g. Belkin et al., 2005; Kahru et al., 2012).

The Fclear effect also adds to the high Fprob values observed during winter. Tidal mixing fronts are absent during this time of the year and the high Fprob indicates, on the one hand, the inclusion of signals from wintertime fronts, which will be discussed in section 4.3. However, the signal was much lower in Fmean. It is likely that higher cloud cover during winter leads to fewer clear pixels and hence, Fvalid being divided by a smaller number of Fclear, which resulted in an elevated Fprob, while Fmean was not affected by the divisor effect.

The relationship between Fprob and Fclear has largely been ignored in the majority of research that uses satellite imagery to investigate temporal variability of fronts (e.g. Belkin et al., 2005; Kahru et al., 2012) and only been mentioned in a couple of studies (Obenour, 2013; Oram et al. 2008; Ullman et al., 2007). Oram et al. 2008 note that the increase in available satellite images during the second half of their study (1997-2002) caused bias in their detection probabilities (Fprob). Ullman et al. (2007) suggested that the non-linear relationship between clear and front pixels is caused by the failure of the SIED-algorithm to identify all frontal pixels as such, particularly in partially cloud-covered scenes. The clouds block the 
contour-following part of the SIED algorithm, resulting in Fprob being underestimated. Obenour (2013) suggests the SIED-window should be at least $90 \%$ cloud-free during image processing in order to avoid exactly this problem and subsequently, avoid temporal variability of Fprob caused by the fraction of clear pixels. Obenour (2013) addresses the Fclear effect by increasing data quality at the expense of data quantity: that approach differs to the one used in this study, which accounts for the amount of clear pixels during the statistical analysis stage, regardless of the difficulties caused by partially cloudy scenes.

Most temporal variability studies focus on seasonal variability and did not report any discontinuities of Fprob caused by Fclear (e.g. Castelao et al., 2014; Hickox et al., 2000; Mavor et al., 2001). However, the Fclear effect appears to be less obvious when investigating seasonal variability, as seen in this study. Less research has focused on interannual patterns and mostly reported an increase in Fprob over time. For example, Belkin and Cornillon (2005) found a surprising 50\% rise in the annual mean of Fprob between 1985-96, averaged over the entire Bering Sea. Similarly, Kahru et al. (2012) showed a significant increase in Fprob in the California Current System over 29 years (1981-2009). However, both studies did not consider the changes in available data. Ullman et al. (2007) used frontal maps from 1985 to 2001 to investigate temporal and spatial variability of Fprob in four regions of the North Atlantic. They mentioned the dependency of Fprob on Fclear, which could lead to an underestimation of Fprob. However, they concluded that it did not influence their results, because seasonal peaks of Fclear did not coincide with peaks in Fprob. In this research the seasonal pattern between Fprob and Flcear were not identical either, showing different seasonal peaks, but the relationship became evident only during the modelling process. Therefore, Ullman et al. (2007) might have underestimated the effect of Fclear. Obenour (2013) is the only study to our knowledge that accounts for the clear pixel issue in their analyses, using the method described above (SIED-window $>90 \%$ cloud free). Despite accounting for Fclear, Obenour (2013) still found an overall increase in global Fprob from 1981 to 2011, which varied between different (selected) regions of the world.

Although most of these studies did not account for Fclear, they generally report a rise in Fprob over time. Direct comparisons between this study and previous research are difficult, because of different study locations (e.g. California Current System, Bering Sea), study periods and durations, and the fact that these studies combine distinct fronts by spatially averaging over large areas. Subsequently, winter and summer time fronts, which may have different long-term trend pattern, are merged. For example, Belkin and Cornillon (2005) use frontal maps from before 1995, a period when the increase in satellite imagery was not as marked. It is possible that a divisor effect in other parts of the world is not as significant 
because of different weather patterns and cloud cover throughout the year. It is also possible that in this research the effect of Fclear has been overestimated by the statistical model, masking genuine temporal variability in the other metrics.

In summary, the effect of Flcear on Fprob and Fmean is strong and the amount of available data should always be considered in any analysis. Because of the non-linear relationship between Fclear and Fprob/Fmean, not all variability will be removed when accounting for Fclear and variability relating to actual changes in frontal occurrence can still be observed. In addition, Fclear is mostly an issue in the lower value ranges. Therefore, one could use data above a certain Fclear threshold only (determined via statistical analysis on the given dataset) and make the assumption that all the variability observed is actually due to changes in the frontal structure. It clearly requires more investigations on how to best account for an Fclear effect. A combined approach appears sensible, whereby an Fclear effect is reduced during frontal map processing (Obenour, 2013) and subsequently, tested for during statistical analysis (this research).

\subsection{Importance of differentiating between distinct types of fronts}

High values of Fprob were found during winter at the Celtic Sea Front, which were likely frontal segments not belonging to the front of interest, but to a coastal current. The inclusion of this signal affects the results of temporal analyses, because it adds variability independent of the front of interest. Different types of fronts respond to atmospheric and hydrodynamic forcing in specific ways and subsequently, display a distinct spatio-temporal variability (Hickox et al., 2000). When summarising frontal activity over large areas, e.g. entire seas, fronts with different temporal variability pattern will be combined and their individual temporal signals blurred. Therefore, it is difficult to draw meaningful conclusions about frontal activity from a cumulative temporal signal obtained over large areas.

It would make sense for any type of temporal analyses, seasonal or trend, to separate distinct types of fronts. In addition, individual fronts or particular types often play a specific role in oceanographic or biological processes and their effect on the ecosystem can vary (Scales et al., 2014). It is therefore of interest for ecologists and oceanographers alike to be able to distinguish between individual features and study them in isolation. Isolating features of interest is difficult, particularly in areas with high frontal activity, where various fronts exist in close proximity and often merge, such as shelf-seas (Achta et al 2015). In this research, the study area was refined by resampling different sized subsets (see supplement 8.1). Although the process was parameterized as much as possible, there is some arbitrariness and the 
possibility of unwanted features entering the study region. A newly developed technique, called synoptic front maps, could prove useful for isolating fronts for analysis. It is based on a novel line-clustering algorithm, which first involves smoothing the Fmean map with a Gaussian, then the most prominent frontal observations and directions are identified and followed to generate contiguous contours. This front simplification algorithm is in preparation for publication (Miller, in preparation).

\section{Conclusions}

Frontal maps were initially developed to visualise fronts, using image processing algorithms to detect, identify and enhance frontal features. However, for statistical analysis the user should be aware of their qualities and limitations. This guide on frontal metrics highlights essential points to think about before and during the analysis stage. Metrics belonging to the group Fprob, Fpers, Fcomp were highly correlated, whereas Fmean and Fdens displayed weaker correlations with other metrics. We recommend the combined use of Fprob and Fmean to thoroughly describe temporal variability of frontal features. The more complex metrics hinder interpretation without adding information. However, for visual analysis, frontal maps based on complex metrics (e.g. Fdens, Fcomp) may be more appropriate, because they highlight persistent features and suppress transient segments that add noise to the maps. Although this appears to make the use of complex metrics in spatial analysis more desirable, e.g. in ecology to explain animal distribution, we still recommend the use of interpretable metrics such as Fprob and Fmean. Alternatively, a combination of metrics (complex, but spatially clean versus simple and noisy, but interpretable) can be used to entangle the relationship between fronts and animal distribution. Secondly, data quantity has to be accounted for as it can introduce spurious trends: Fprob and Fmean were strongly affected by Fclear. A combination of improving data quality during the data processing stage as well as including Fclear as a factor in statistical models is recommended. We used frontal maps at monthly resolution and focused on a specific type of front in this research. It would be useful to investigate the Fclear effect on fronts in other regions, on other types of fronts and at higher temporal resolutions. For example, frontal types other than tidal mixing fronts, which are not subject to meteorological factors (which tends to covary with Fclear) as much could be less sensitive to Fclear. Finally, depending on the research question, scientists should consider studying individual fronts in isolation to avoid blurring of signals due to contrasting temporal food prints of different frontal types. 


\section{Acknowledgements}

We thank the University of Southampton for financial support of this research and NEODAAS for acquisition and processing of the satellite data. We are also grateful for advice and comments from scientists in the Physical Oceanography group of the University of Plymouth and Dr. Claire Embling and Dr. Simon Ingram from the Plymouth Marine Science Department. We would like to thank five anonymous reviewers and Dr. Herve Demarcq from IRD Sete for their constructive comments, which greatly improved the manuscript and the editor in chief of Deep Sea Research Part I, Dr. Igor Belkin. 


\section{References}

Acha, E. M., Piola, A., Iribarne, O., \& Mianzan, H. (2015). Ecological Processes at Marine Fronts: Oases in the Ocean: Springer.

Armstrong, E. M., Wagner, G., Vazquez-Cuervo, J., \& Chin, T. M. (2012). Comparisons of regional satellite sea surface temperature gradients derived from MODIS and AVHRR sensors. International Journal of Remote Sensing, 33(21), 6639-6651. doi: 10.1080/01431161.2012.692832

Bauer, R. K., Fromentin, J. M., Demarcq, H., Brisset, B., \& Bonhommeau, S. (2015). Co occurrence and habitat use of fin whales, striped dolphins and Atlantic Bluefin tuna in the Northwestern Mediterranean Sea. PloS one, 10(10), e0139218.

Belkin, I. M., \& Cornillon, P. (2003). SST fronts of the Pacific coastal and marginal seas. Pacific Oceanography, 1(2), 90-113.

Belkin, I. M., \& Cornillon, P. C. (2005). Bering Sea thermal front from Pathfinder data: Seasonal and interannual variability. Pacific Oceanography, 2(3-4), 6-20.

Belkin, I. M., Cornillon, P. C., \& Sherman, K. (2009). Fronts in Large Marine Ecosystems. Progress in Oceanography, 81(1-4), 223-236. doi: 10.1016/j.pocean.2009.04.015

Brodie, S., Hobday, A. J., Smith, J. A., Everett, J. D., Taylor, M. D., Gray, C. A., \& Suthers, I. M. (2015). Modelling the oceanic habitats of two pelagic species using recreational fisheries data. Fisheries oceanography, 24(5), 463-477.

Brown, J., Carrillo, L., Fernand, L., Horsburgh, K. J., Hill, A. E., Young, E. F., \& Medler, K. J. (2003). Observations of the physical structure and seasonal jet-like circulation of the Celtic Sea and St. George's Channel of the Irish Sea. Continental Shelf Research, 23(6), 533-561. doi: 10.1016/s0278-4343(03)00008-6

Castelao, R. M., \& Wang, Y. T. (2014). Wind-driven variability in sea surface temperature front distribution in the California Current System. Journal of Geophysical ResearchOceans, 119(3), 1861-1875.

Cayula, J. F., \& Cornillon, P. (1992). Edge-detection algorithm for SST images. Journal of Atmospheric and Oceanic Technology, 9(1), 67-80. doi: 10.1175/15200426(1992)009<0067:edafsi>2.0.co;2

Cyr, F., \& Larouche, P. (2015). Thermal Fronts Atlas of Canadian Coastal Waters. Atmosphere-Ocean, 53(2), 212-236. doi: 10.1080/07055900.2014.986710

Defra. (2009). Guidance note on "Selection and designation of Marine Conservation Zones" (Note 1). Published by the Department for Environment, Food and Rural Affairs.

Defra. (2012). "Marine Strategy Framework Directive Consultation: UK initial assessment and proposals for Good Environmental Status": London: Department for Environment, Food and Rural Affairs.

Doniol-Valcroze, T., Berteaux, D., Larouche, P., \& Sears, R. (2007). Influence of thermal fronts on habitat selection by four rorqual whale species in the Gulf of St. Lawrence. Marine Ecology-Progress Series, 335, 207-216. 
Edwards, E. W. J., Quinn, L. R., Wakefield, E. D., Miller, P. I., \& Thompson, P. M. (2013). Tracking a northern fulmar from a Scottish nesting site to the Charlie-Gibbs Fracture Zone: Evidence of linkage between coastal breeding seabirds and Mid-Atlantic Ridge feeding sites. Deep-Sea Research Part Ii-Topical Studies in Oceanography, 98, 438444. doi: $10.1016 /$ j.dsr2.2013.04.011

Elliott, A. J., \& Clarke, T. (1991). Seasonal stratification in the Northwest European shelfseas. Continental Shelf Research, 11(5), 467-492. doi: 10.1016/0278-4343(91)90054a.

European Union. (2008). Directive 2008/56/EC of the European Parliament and the Council of 17 June 2008. Establishing a framework for community action in the field of marine environmental policy (Marine Strategy Framework Directive). . Official Journal of the European Union, L164, 19-40.

Hastie, T., \& Tibshirani, R. (1987). Generalized additive models- Some applications. Journal of the American Statistical Association, 82(398), 371-386. doi: 10.2307/2289439

Hickox, R., Belkin, I., Cornillon, P., \& Shan, Z. (2000). Climatology and seasonal variability of ocean fronts in the east China, Yellow and Bohai Seas from satellite SST data. Geophysical Research Letters, 27(18), 2945-2948. doi: 10.1029/1999gl011223

Holt, J., \& Umlauf, L. (2008). Modelling the tidal mixing fronts and seasonal stratification of the Northwest European Continental shelf. Continental Shelf Research, 28(7), 887903. doi: 10.1016/j.csr.2008.01.012

Holt, J., Wakelin, S., Lowe, J., \& Tinker, J. (2010). The potential impacts of climate change on the hydrography of the northwest European continental shelf. Progress in Oceanography, 86(3-4), 361-379. doi: 10.1016/j.pocean.2010.05.003

Hopkins, J., Shaw, A. G. P., \& Challenor, P. (2010). The Southland front, New Zealand: variability and ENSO correlations. Continental Shelf Research, 30(14), 1535-1548

Horsburgh, K. J., Hill, A. E., \& Brown, J. (1998). A summer jet in the St George's Channel of the Irish Sea. Estuarine Coastal and Shelf Science, 47(3), 285-294. doi: 10.1006/ecss.1998.0354

Kahru, M., Di Lorenzo, E., Manzano-Sarabia, M., \& Mitchell, B. G. (2012). Spatial and temporal statistics of sea surface temperature and chlorophyll fronts in the California Current. Journal of Plankton Research, 34(9), 749-760. doi: 10.1093/plankt/fbs010

Lee, M. A., Chang, Y., \& Shimada, T. (2015). Seasonal evolution of fine-scale sea surface temperature fronts in the East China Sea. Deep-Sea Research Part Ii-Topical Studies in Oceanography, 119, 20-29. doi: 10.1016/j.dsr2.2014.03.021

LeFevre, J. (1986). Aspects of the biology of frontal systems. Advances in Marine Biology, 23, 163-299.

Marsh, R., Hickman, A. E., \& Sharples, J. (2015). S2P3-R (v1.0): a framework for efficient regional modelling of physical and biological structures and processes in shelf-seas. Geoscientific Model Development, 8(10), 3163-3178. doi: 10.5194/gmd-8-3163-2015 
Mavor, T. P., \& Bisagni, J. J. (2001). Seasonal variability of sea-surface temperature fronts on Georges Bank. Deep-Sea Research Part Ii-Topical Studies in Oceanography, 48(1-3), 215-243. doi: 10.1016/s0967-0645(00)00120-x

McClatchie, S., Cowen, R., Nieto, K., Greer, A., Luo, J. Y., Guigand, C. \& Rudnick, D. (2012). Resolution of fine biological structure including small narcomedusae across a front in the Southern California Bight. Journal of Geophysical Research: Oceans, $117(\mathrm{C} 4)$

Miller, P. (2009). Composite front maps for improved visibility of dynamic sea-surface features on cloudy SeaWiFS and AVHRR data. Journal of Marine Systems, 78(3), 327-336. doi: 10.1016/j.jmarsys.2008.11.019

Miller, P. I. (in preparation). A line clustering algorithm with application to simplifying ocean front maps derived from satellite data. . Remote Sensing of Environment.

Neil, C., Cunningham, A., McKee, D., \& Polton, J. A. (2012). Remote sensing of seasonal stratification dynamics in the southern Irish Sea. Remote Sensing of Environment, 127, 288-297. doi: 10.1016/j.rse.2012.09.010

Nieblas, A. E., Demarcq, H., Drushka, K., Sloyan, B., \& Bonhommeau, S. (2014). Front variability and surface ocean features of the presumed southern bluefin tuna spawning grounds in the tropical southeast Indian Ocean. Deep Sea Research Part II: Topical Studies in Oceanography, 107, 64-76.

Nieto, K., Demarcq, H., \& McClatchie, S. (2012). Mesoscale frontal structures in the Canary Upwelling System: New front and filament detection algorithms applied to spatial and temporal patterns. Remote Sensing of Environment, 123, 339-346.

Obenour, K. M. (2013). Temporal trends in global sea surface temperature fronts (Master's Thesis). Retrieved from http://digitalcommons.uri.edu/theses/136 (Paper 136)

Oram, J. J., McWilliams, J. C., \& Stolzenbach, K. D. (2008). Gradient-based edge detection and feature classification of sea-surface images of the Southern California Bight. Remote Sensing of Environment, 112(5), 2397-2415.

Owen, R. (Ed.). (1981). Fronts and Eddies in the Sea: mechanisms, interactions and biological effects. Analysis of marine ecosystems, 197-233. New York: Academic Press.

Park, K. A., Ullman, D. S., Kim, K., Chung, J. Y., \& Kim, K. R. (2007). Spatial and temporal variability of satellite-observed subpolar front in the East/Japan Sea. Deep Sea Research Part I: Oceanographic Research Papers, 54(4), 453-470

Peng, R. D. (2008). R package 'simpleboot': Simple Bootstrap Routines (version 1.1-3).

Pingree, R. D. (1975). Advance and retreat of the thermocline on the continental shelf. Journal of the Marine Biological Association of the United Kingdom, 55(4), 965-974.

Pingree, R. D., \& Griffiths, D. K. (1978). Tidal fronts on shelf-seas around British Isles. Journal of Geophysical Research-Oceans and Atmospheres, 83(NC9), 4615-4622.

Pirotta, E., Thompson, P. M., Miller, P. I., Brookes, K. L., Cheney, B., Barton, T. R., Graham, I. M., \& Lusseau, D. (2014). Scale-dependent foraging ecology of a marine 
top predator modelled using passive acoustic data. Functional Ecology, 28(1), 206217. doi: $10.1111 / 1365-2435.12146$

Priede, I. G., \& Miller, P. I. (2009). A basking shark (Cetorhinus maximus) tracked by satellite together with simultaneous remote sensing II: New analysis reveals orientation to a thermal front. Fisheries Research (Amsterdam), 95(2-3), 370-372. doi: :10.1016/j.fishres.2008.09.03

Roa-Pascuali, L., Demarcq, H., \& Nieblas, A. E. (2015). Detection of mesoscale thermal fronts from $4 \mathrm{~km}$ data using smoothing techniques: Gradient-based fronts classification and basin scale application. Remote Sensing of Environment, 164, 225237.

Scales, K. L., Miller, P. I., Hawkes, L. A., Ingram, S. N., Sims, D. W., \& Votier, S. C. (2014). On the Front Line: frontal zones as priority at-sea conservation areas for mobile marine vertebrates. Journal of Applied Ecology, 51(6), 1575-1583. doi: $10.1111 / 1365-2664.12330$

Scales, K. L., Miller, P. I., Varo-Cruz, N., Hodgson, D. J., Hawkes, L. A., \& Godley, B. J. (2015). Oceanic loggerhead turtles Caretta caretta associate with thermal fronts: evidence from the Canary Current Large Marine Ecosystem. Marine Ecology Progress Series, 519, 195-207. doi: 10.3354/meps11075

Shimada, T., Sakaida, F., Kawamura, H., \& Okumura, T. (2005). Application of an edge detection method to satellite images for distinguishing sea surface temperature fronts near the Japanese coast. Remote sensing of environment, 98(1), 21-34.

Simpson, J. H., Crisp, D. J., \& Hearn, C. (1981). The shelf-sea fronts: implications of their existence and behaviour [and discussion]. Philosophical Transactions of the Royal Society of London A: Mathematical, Physical and Engineering Sciences, 302: 531-546.

Simpson, J. H., \& Sharples, J. (2012). Introduction to the physical and biological oceanography of shelf-seas: Cambridge University Press.

Suberg, L. A. (2015). Investigations of the variability of tidal mixing fronts and their importance for shelf-sea ecosystems across multiple trophic levels (Unpublished doctoral thesis). University of Southampton, Faculty of Natural and Environmental Sciences, Southampton, UK.

Ullman, D. S., Cornillon, P. C., \& Shan, Z. (2007). On the characteristics of subtropical fronts in the North Atlantic. Journal of Geophysical Research-Oceans, 112(C1). doi: C0101010.1029/2006jc003601

Wingfield, D. K., Peckham, S. H., Foley, D. G., Palacios, D. M., Lavaniegos, B. E., Durazo, R. \& Bograd, S. J. (2011). The making of a productivity hotspot in the coastal ocean. PloS one, 6(11), e27874.

Wood, S. N. (2006). Generalized Additive Models: An Introduction with R. Chapman and Hall/CRC.

Xu, Y., Nieto, K., Teo, S. L., McClatchie, S., \& Holmes, J. (2017). Influence of fronts on the spatial distribution of albacore tuna (Thunnus alalunga) in the Northeast Pacific over the past 30 years (1982-2011). Progress in Oceanography, 150, 72-78. 
Young, E. F., Brown, J., Aldridge, J. N., Horsburgh, K. J., \& Fernand, L. (2004). Development and application of a three-dimensional baroclinic model to the study of the seasonal circulation in the Celtic Sea. Continental Shelf Research, 24(1), 13-36. doi: 10.1016/j.csr.2003.09.003 